\title{
Administrativer Aufwand für Ärzte steigt weiter an
}

\section{Beatrix Meyera, Barbara Rohner ${ }^{b}$, Lukas Golder ${ }^{c}$, Claude Longchamp $^{d}$ \\ ${ }^{a}$ Leiterin Abteilung Tarife und Gesundheitsökonomie Spitalärzte; ${ }^{b}$ Wissenschaftliche Mitarbeiterin Abteilung Tarife und Gesundheitsökonomie Spitalärzte; \\ ${ }^{c}$ Senior-Projektleiter, gfs.bern; ${ }^{d}$ Institutsleiter, gfs.bern}

Nur noch rund ein Drittel ihrer Arbeitszeit wenden Ärztinnen und Ärzte der Akutsomatik für patientennahe Tätigkeiten auf, in der Psychiatrie und der Rehabilitation sogar nur etwa ein Viertel. Einen grossen Teil des Tages verbringen die Ärzte mit administrativen Arbeiten; die Assistenzärzte sind dabei besonders betroffen.

Seit vier Jahren rechnen die Spitäler in der Akutsomatik mit dem Tarifsystem SwissDRG ab. Per Januar 2018 ist geplant, dass zwei neue stationäre Tarifsysteme hinzukommen - TARPSY für die Psychiatrie und ST Reha für die Rehabilitation. Wie hat sich der Alltag in der Akutsomatik verändert und wie sind die betroffenen Ärztinnen und Ärzte gegenüber der Einführung von TARPSY und ST Reha eingestellt? Um allfällige Fehlentwicklungen frühzeitig feststellen zu können, führt gfs.bern im Auftrag der FMH seit 2011 jährlich wiederkehrende repräsentative Befragungen der Ärzteschaft durch [1].

\section{Sinkender Anteil an patientennahen Tätigkeiten}

Viele Ärztinnen und Ärzte dürften ihren Beruf gewählt haben, um möglichst für ihre Patienten da sein zu können. Seit 2011 hat jedoch der Anteil der medizinischen patientennahen Tätigkeiten der Spitalärzte sowohl in der Akutsomatik als auch in der Psychiatrie und Rehabilitation klar abgenommen. Die Akutsomatiker verbrachten rund einen Drittel ihrer Zeit mit patientennahen Tätigkeiten, die in der Psychiatrie und in der Rehabilitation tätigen Ärzte sogar nur noch rund einen Viertel (Tab. 1).

Tabelle 1: Vergleich Zeitaufwand für verschiedene Tätigkeiten.

«Wenn Sie an den letzten normalen Arbeitstag denken, wie viel Zeit haben Sie dabei auf die folgenden Tätigkeiten angewendet? Bitte geben Sie Ihre Angaben in Minuten an.» (In \% Mittelwerte einzelner Tätigkeiten am gesamten Arbeitstag).

\begin{tabular}{|c|c|c|c|c|}
\hline & $\begin{array}{l}\text { akutsomatische } \\
\text { Ärzte/Ärztinnen }\end{array}$ & $\begin{array}{l}\text { akutsomatische Assis- } \\
\text { tenzärzte/-ärztinnen }\end{array}$ & Psychiatrie & Rehabilitation \\
\hline Medizinische patientennahe Tätigkeiten & 35,1 & 29,1 & 27,2 & 22,9 \\
\hline Visiten & 9,5 & 10,2 & 8,2 & 14,8 \\
\hline $\begin{array}{l}\text { Rapporte / medizinischer Informationsaustausch / } \\
\text { Fallbesprechungen }\end{array}$ & 9,6 & 10,2 & 10,7 & 8,1 \\
\hline Ärztliche Dokumentationsarbeit / Patientendossier & 16,9 & 27,3 & 10,8 & 18,3 \\
\hline Kodierung erbrachter Leistungen & 1,9 & 1,8 & 2,9 & 2,3 \\
\hline Überprüfung von Kodierung & 0,6 & 0,3 & 1,3 & 0,9 \\
\hline $\begin{array}{l}\text { Anfragen von Krankenkassen beantworten / } \\
\text { administrativer Verkehr mit den Versicherungen }\end{array}$ & 2,3 & 2,3 & 3,6 & 3,7 \\
\hline Kostengutsprachen einholen & 0,9 & 1,3 & 1,9 & 3,0 \\
\hline Organisation der Nachbehandlung & 2,4 & 3,3 & 2,5 & 2,8 \\
\hline $\begin{array}{l}\text { Ohne Bezug zu Patiententätigkeit: Organisatorisches } \\
\text { in der Klinik / Korrespondenz }\end{array}$ & 7,1 & 3,9 & 10,4 & 9,7 \\
\hline Sonstige administrative Tätigkeiten & 4,6 & 3,5 & 6,5 & 5,2 \\
\hline Aus- und Weiterbildung, Ausbildung KollegInnen, Literaturstudium & 5,4 & 4,3 & 6,5 & 5,5 \\
\hline Forschungsarbeiten & 1,5 & 1,1 & 1,6 & 0,9 \\
\hline Sonstige nicht-medizinische Tätigkeiten & 2,1 & 1,4 & 3,8 & 2,0 \\
\hline $\begin{array}{l}\text { Umsetzungsarbeiten / Tätigkeiten aufgrund des neuen Kinder- } \\
\text { und Erwachsenenschutzrechts }\end{array}$ & - & - & 2,1 & - \\
\hline
\end{tabular}

(c) gfs.bern, Begleituntersuchung SwissDRG, ST Reha, TARPSY im Auftrag der FMH, Juni-August 2015 
Dafür hat der administrative Aufwand im Spital weiter zugenommen. Der Anteil des Dokumentationsaufwands für das Patientendossier in der Akutsomatik und in der Rehabilitation ist über die vergangenen Jahre angestiegen. In der Akutsomatik verbrachten die Ärztinnen und Ärzte im Jahr 2015 durchschnittlich rund 15 Minuten mehr Zeit pro Tag mit Dokumentationsarbeit als 2011. Betroffen sind dabei vor allem die Assistenzärzte: Im Jahr 2015 verwendeten sie 27\% ihrer Arbeitszeit für Dokumentationsarbeiten. In der Psychiatrie ist der Dokumentationsaufwand für das Patientendossier zwar am geringsten, dafür verursacht dort das neue Kinder- und Erwachsenenschutzrecht zunehmenden administrativen Aufwand (Tab. 1).

\section{Hohe Belastung aufgrund von Zeitdruck und Stress}

Eine klare Mehrheit der Ärztinnen und Ärzte fühlt sich andauernd hohem Zeitdruck ausgesetzt. Eine zusätzliche Belastung stellen die Überstunden dar. Am meisten Überstunden leistet die akutsomatisch tätige Spitalärzteschaft mit durchschnittlich 7,4 Überstunden pro Woche. Die Spitalärzte der Rehabilitation leisten 6,4, die Psychiater 5,4 und die praxisambulanten Ärzte 5,8 Überstunden pro Woche.

Diese konstante Überbelastung der Ärzteschaft wirkt sich negativ auf ihr Befinden aus. Heute beklagen sich

\section{Gute Teamarbeit trotz zunehmendem Stress.}

rund 50\% der Spitalärzte sowie rund $40 \%$ der praxisambulant tätigen Ärzte darüber, meistens oder häufig unter Stress zu leiden. Der Stress nahm im Vergleich zu 2013 bei allen Ärztegruppen zu, am meisten jedoch bei den Psychiatern.

\section{Ärzte trotzdem zufrieden mit ihrer Tätigkeit}

Trotz hoher Arbeitsbelastung ist die Mehrheit der Ärztinnen und Ärzte sehr oder eher zufrieden mit ihrer Arbeitstätigkeit. Dies dürfte unter anderem mit der hohen Berufsidentifikation und der guten Zusammenarbeit im Team zusammenhängen. Rund 95\% der Spitalärzte sind zufrieden oder eher zufrieden mit ihrem Team. 90\% der in der Akutsomatik tätigen Spitalärzte finden die Zusammenarbeit mit der Pflege sowie 77\% die Zusammenarbeit mit praxisambulant tätigen Ärzten sehr gut oder gut. $49 \%$ bewerten die Zusammenarbeit mit der Spitalverwaltung und 29\% die Zusammenarbeit mit den Versicherungen als sehr gut oder gut eine leichte Verbesserung im Vergleich zu den Vorjah- ren. Die Strategie ihres Spitals schätzen knapp 70\% der akutsomatisch tätigen Ärztinnen und Ärzte als sehr gut oder gut ein, in der Psychiatrie und Rehabilitation sind es über $80 \%$. Konstante $10 \%$ der Spitalärzte denken jedoch über eine Stelle ausserhalb des Gesundheitswesens nach, während es bei den praxisambulant tätigen Ärzten $6 \%$ sind.

\section{Vorbehalte insbesondere gegenüber TARPSY}

Grosse Vorbehalte haben die Psychiaterinnen und Psychiater im Hinblick auf die Einführung von TARPSY: Über die Hälfte steht der Einführung klar oder eher ablehnend gegenüber. In der Rehabilitation haben rund 30\% eine klar oder eher ablehnende Haltung gegenüber ST Reha. Die grösseren Vorbehalte in der Psychiatrie könnten damit zusammenhängen, dass bei TARPSY über einen Fallpauschalenanteil diskutiert wird und bei ST Reha dieser nicht vorgesehen ist.

\section{Gute Patientenversorgung trotz einiger Fehlentwicklungen}

Eine klare Mehrheit der befragten Spitalärzteschaft schätzt den Versorgungsstandard in ihrem unmittelbaren Arbeitsumfeld als sehr gut oder eher gut ein. Den Spielraum für die Art und Weise der Behandlung erachten rund $75 \%$ der akutsomatisch tätigen Spitalärztinnen und -ärzte als sehr hoch oder hoch. Praktisch konstante 15\% der Akutsomatiker geben jedoch an, dass die Krankenkassen einen Einfluss auf die Behandlung nehmen. Analog zu 2013 stellen die Akutsomatiker durchschnittlich rund fünfmal pro Monat fest, dass Krankenkassen Kostengutsprachen für die Rehabilitation verzögern. Knapp 50\% der in der Rehabilitation sowie $10 \%$ der akutsomatisch tätigen Spitalärzte sind der Ansicht, dass Überweisungen in die Rehabilitation zu früh erfolgten. Weiterhin kritischer als vor Einführung von SwissDRG beurteilen die praxisambulant tätigen Ärzte den Entlassungszeitpunkt der ihnen zugewiesenen Patienten aus den Spitälern - für über 20\% erfolgt dieser häufig zu früh. Rund 20\% der Spitalärzte beobachteten, dass versucht wird, chronisch Kranke in andere Einrichtungen zu verschieben.

\section{Bonuszahlungen zunehmend verbreitet}

In den Schweizer Spitälern erhalten rund ein Viertel der Ärztinnen und Ärzte eine Bezahlung mit einem variablen Lohnbestandteil. Es handelt sich dabei um Bonuszahlungen aufgrund von Zielvereinbarungen oder andere variable Lohnkomponenten wie zum Bei- 
Frage 1 «Existieren auf Ihrer Abteilung / in Ihrer Klinik Entschädigungssysteme mit variablen, leistungsabhängigen Lohnkomponenten?»

Frage 2 «Enthält Ihr persönliches Entschädigungssystem eine variable, leistungsabhängige Lohnkomponente?» Frage 3 (falls variable, leistungsabhängige Lohnkomponente) «Handelt es sich bei der variablen, leistungsabhängigen Lohnkomponente um:»

in \% akutsomatische Ärzte

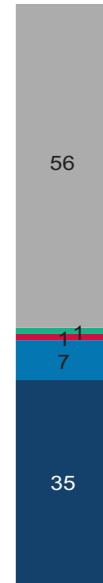

Assistenzarzt/-ärztin
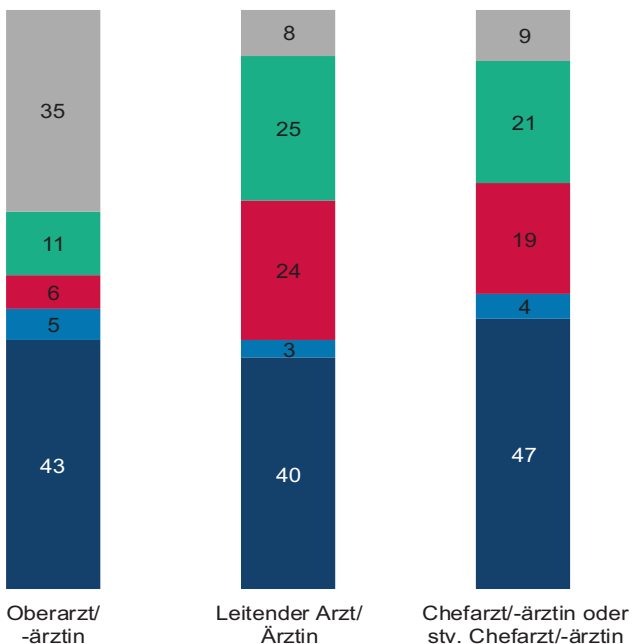

- gfs bern, Begleituntersuchung SwissDRG, ST Reha, TARPSY im Auftrag der FMH, Juni - August 2015

weiss nicht/keine Antwort bei Fragen 1, 2 oder 3

ausschliesslich andere leistungsabhängige Lohnkomponenten ohne Bonuszahlungen

- Bonuszahlungen (teilweise kombiniert mit anderen leistungsabhängigen Lohnkomponenten)

- keine persönliche leistungsabhängige

Lohnkomponente

- keine leistungsabhängige Lohnkomponente in Abteilung/Klinik

(c) gfs.bern, Begleituntersuchung
(N akutsomatische Ärzte $=804$ )

Abbildung 1: Leistungsabhängige Lohnkomponenten der akutsomatisch tätigen Spitalärzte nach Position.

spiel privatärztliche Honorare. 12\% der akutsomatisch tätigen Spitalärzte, 10\% der Psychiater und 14\% der in der Rehabilitation tätigen Spitalärzte beziehen einen Bonus. In der Akutsomatik hat speziell auf Stufe der leitenden Spitalärzte der Anteil der Bonusbezüger zugenommen und beträgt bereits $24 \%$. Bei den Chefärzten erhalten 19\% einen Bonus (vgl. Abb. 1).

Der Anteil des leistungsabhängigen Bonus an der gesamten Lohnsumme nahm im Vergleich zu den beiden Vorjahren insbesondere bei den Chef- und Oberärzten deutlich zu. So stieg dieser bei den Chefärzten

\section{Praxisambulant tätige Ärzte beurteilen Entlassungszeitpunkt der Patienten aus Spitälern kritischer.}

von durchschnittlich 19\% im Jahr 2012 auf 26\% im Jahr 2014 und bei den Oberärzten von 8\% auf 16\%. Auf Stufe Leitender Arzt stieg der Anteil leicht an auf 26\%. Problematisch ist die Situation, wenn Bonuszahlungen an die Anzahl Operationen geknüpft sind und so eine Mengenausweitung auslösen können [2]. Die im Jahr 2013 befragten akutsomatisch tätigen Ärzte haben während der letzten 30 Tage im Schnitt 0,6 Operationen beobachtet, die nicht medizinisch indiziert waren; Im Jahr 2015 stiegen diese leicht an auf 0,9. Den weiteren Verlauf der Anzahl nicht medizinisch indizierter Operationen gilt es kritisch zu verfolgen.

\section{Entwicklung beobachten - Prozesse verbessern}

Den Trend gilt es zu stoppen, dass die Spitalärztinnen und -ärzte zunehmend mit administrativen Arbeiten belastet werden und der Anteil an medizinischen patientennahen Tätigkeiten weiter abnimmt. Die Ärzteschaft kann nicht alle Probleme mit Überstunden auffangen, weshalb es mittelfristig Veränderungen braucht. Abgesehen davon könnte sich der zunehmende Stress und Zeitdruck auf die Behandlungsqualität auswirken. Bereits heute haben rund ein Drittel der befragten Ärztinnen und Ärzte den Eindruck, dass aufgrund der hohen Arbeitsbelastung und des Zeitdrucks die Qualität der Patientenversorgung beeinträchtigt wird. Es ist zu hoffen, dass die Spitäler ihre Prozesse stetig weiter verbessern. Erste positive Anzeichen gibt es in der Akutsomatik bereits: 30\% der Befragten gaben an, dass sie sich dank effizienter Prozesse besser auf ihre medizinische Tätigkeit konzentrieren können - im Jahr 2013 waren es erst $23 \%$ gewesen.

\section{Referenzen}

1 Zur ausführlichen Studie vgl. Golder et al. Trotz steigendem Dossieraufwand bleibt die Spitalärzteschaft motiviert. Schlussbericht 2015. www.fmh.ch $\rightarrow$ Stationäre Tarife $\rightarrow$ Begleitforschung $\rightarrow$ Entwicklung Rahmenbedingungen Ärzteschaft.

2 Vgl. FMH-Positionspapier zu Bonusvereinbarungen in Spitalarztverträgen. www.fmh.ch $\rightarrow$ Stationäre Tarife $\rightarrow$ Positionen $\rightarrow$ Positionspapiere $\rightarrow$ Positionspapier der FMH zu Bonusvereinbarungen in Spitalarztverträgen. 